IdeAs

Idées d'Amériques

Poètes et éditeurs : diffuser la poésie d'avant-garde américaine (depuis 1945)

\title{
Les réactions au Québec et au Canada au discours du général de Gaulle de 1967
}

\section{Stéphane Paquin}

\section{(2) OpenEdition}

\section{Journals}

Édition électronique

URL : https://journals.openedition.org/ideas/1912

DOI : $10.4000 /$ ideas. 1912

ISSN : 1950-5701

Éditeur

Institut des Amériques

Référence électronique

Stéphane Paquin, «Les réactions au Québec et au Canada au discours du général de Gaulle de 1967 », IdeAs [En ligne], 9 | 2017, mis en ligne le 12 juillet 2017, consulté le 19 octobre 2022. URL : http:// journals.openedition.org/ideas/1912 ; DOI : https://doi.org/10.4000/ideas.1912

Ce document a été généré automatiquement le 19 octobre 2022.

\section{(c)}

Creative Commons - Attribution - Pas d'Utilisation Commerciale - Pas de Modification 4.0 International - CC BY-NC-ND 4.0

https://creativecommons.org/licenses/by-nc-nd/4.0/ 


\title{
Les réactions au Québec et au Canada au discours du général de Gaulle de 1967
}

\author{
Stéphane Paquin
}

1 En 1966, l'Union nationale dirigée par Daniel Johnson prend le pouvoir à Québec. Ce parti nationaliste, à la droite de l'échiquier politique québécois, s'était fait élire en promettant aux Québécois «l'Égalité ou l'Indépendance». L'égalité des francophones dans le Canada dont la seule langue officielle était toujours uniquement l'anglais ou l'indépendance du Québec.

2 La discrimination dont étaient victimes les francophones dans ce pays majoritairement anglophone venait de faire l'objet d'une importante commission d'enquête, la commission d'enquête sur le bilinguisme et le biculturalisme. À la lecture des résultats des travaux de cette commission, il est facile de déduire que les Québécois sont la cible de racisme systémique au Canada. Le contexte des années 1960 au Québec est aussi celui de la Révolution tranquille, révolution sans violence où les francophones du Québec utilisent les leviers de l'état québécois pour se sortir de leur condition d'infériorité politique et économique. Les années 1960 sont également marquées par une vague d'attentats terroristes du Front de libération nationale.

Dans ce contexte politique particulièrement chargé, le premier ministre Daniel Johnson doit être l'hôte, moins d'un an après son élection, de l'Exposition universelle de 1967, exposition à laquelle participeront 44 chefs d'État ou leurs représentants dont la Reine Élizabeth II ou le président des États-Unis Lyndon B. Johnson.À la suite d'une demande du gouvernement du Québec, tous les chefs d'État ont été invités par le gouvernement fédéral à venir au Québec en tant qu'hôtes officiels du gouvernement québécois (Paquin S., 2006). 


\section{La relation France-Québec} Gaulle s'intéresse particulièrement au Québec (Peyrefitte A., 2000). Il avait mandaté André Malraux pour s'occuper de la question québécoise. Rapidement une délégation du Québec, une sorte de mini-ambassade, sera créée à Paris en 1961. Des ententes internationales en matière de culture et d'éducation suivront.

Peu après la prise de pouvoir, le premier ministre du Québec cherche à convaincre le président de Gaulle de venir à l'Expo 1967. Il s'agissait de la troisième invitation que recevait le président pour cet évènement. Le maire de Montréal, Jean Drapeau, ainsi que le Gouverneur général du Canada, Georges Vanier, avaient également invité de Gaulle. Le général de Gaulle avait refusé toutes les invitations.

Il déclare à ce sujet à un de ses conseillers : « Vous ne me voyez quand même pas faire ce déplacement pour aller à la foire » (Thompson D., $1990: 266$ ).De plus, l'invitation de Vanier proposait au Général d'assister aux commémorations du centenaire de la Confédération du Canada. Cette proposition constitue une véritable insulte pour de Gaulle, car, comme il l'écrira: «Nous n'avons pas à féliciter les Canadiens ni nousmêmes de la création d'un "État » fondé sur notre défaite d'autrefois et surtout de l'intégration d'une partie du peuple français dans un ensemble britannique. Au demeurant, cet ensemble est devenu bien précaire »(Thompson D., 1990: 266). Le gouvernement du Québec redoublera d'efforts pour convaincre de Gaulle de changer d'avis.

7 Le général de Gaulle arrive finalement au Québec le 23 juillet à bord d'un navire de guerre, Le Colbert. Plutôt que d'accoster à Montréal, le chef de l'État français descend à Québec pour ensuite se rendre à Montréal en voiture. Entre temps, le président est l'invité de plusieurs réceptions dans la vieille capitale et notamment l'hôte du premier ministre du Québec au Château Frontenac pour un dîner d'État. Lors de ce dîner, le Général déclare: «(...) on assiste ici, comme en maintes régions du monde, à l'avènement d'un peuple qui, dans tous les domaines, veut disposer de lui-même et prendre en main ses destinées. Qui donc pourrait s'étonner ou s'alarmer d'un tel mouvement aussi conforme aux conditions modernes de l'équilibre de notre univers et de l'esprit de notre temps? » (Patry A., 1980 : 102). Le ton était donné.

Le lendemain matin, le président français se rend à Montréal par voiture avec le premier ministre du Québec. Ces derniers empruntent le Chemin du Roy (de France) qui longe le fleuve St-Laurent jusqu'à Montréal et qui a été aménagé pour la circonstance avec des drapeaux français et du Québec.

9 Tout au long de ce trajet, le ton et le langage du Général ne laissent aucun doute sur le fond de sa pensée : le Québec se dirige vers l'autodétermination. À Trois-Rivières, à la suite d'un déjeuner municipal, de Gaulle déclare : «C'est le génie de notre temps que chaque peuple doit disposer de lui-même. Je suis convaincu que c'est ce qui est en train de se passer ici » (Patry A., 1980 : 101). À Montréal, il est reçu par une gigantesque foule et de Gaulle conclura, sur le balcon de l'Hôtel de Ville de Montréal - où il n'était pas prévu qu'il prenne la parole -, par sa tirade célèbre :

Je vais vous confier un secret que vous ne répèterez à personne. Ce soir, ici, et tout le long de ma route, je me suis trouvé dans une atmosphère du même genre que celle de la libération.[...] Vive Montréal ! Vive le Québec ! Vive le Québec libre ! Vive le Canada français! Vive la France! 


\section{Les réactions au Canada et au Québec}

10 Si on avait pris le pouls des Canadiens anglophones après la déclaration du Général sur le balcon de l'Hôtel de Ville, on aurait diagnostiqué l'hypertension. Cette déclaration du président français provoquera l'enthousiasme de la foule et la colère des représentants du gouvernement du Canada qui ne supportent pas cette intrusion dans les affaires intérieures du Canada ainsi que la comparaison indirecte aux nazis. En réponse à ses détracteurs, de Gaulle déclare, le lendemain, lors d'un déjeuner avec le maire Jean Drapeau : «Et quant au reste, tout ce qui grouille, grenouille, scribouille n'a pas plus de conséquences historiques dans ces grandes circonstances qu'il n'en eut jamais dans d'autres» (Patry A., 1980 : 102). De la capitale du Canada, le chef de l'opposition, John Diefenbaker, un anglophone unilingue, est furieux. De nombreux ministres fédéraux haussent le ton. Le Premier ministre du Nouveau-Brunswick, province comportant une importante minorité francophone, Louis Robichaud, annule son voyage à Montréal en guise de protestation.

11 Le Premier ministre du Canada, Lester B. Pearson (qui a obtenu le prix Nobel de la Paix en 1957 pour avoir contribué à l'établissement de la force de paix de l'ONU après la crise de Suez), se résigne à émettre un communiqué officiel pour dénoncer la déclaration du président français. Le communiqué du gouvernement fédéral reste très mesuré compte tenu des circonstances parce que Pearson était conscient de l'attachement des Québécois à la France et à de Gaulle. Il est conscient également de l'effervescence politique au Québec. Le communiqué, qui sera lu lors d'une conférence de presse télévisée, indique que les déclarations du président sont « inacceptables pour le peuple canadien et son gouvernement». Le Premier ministre ajoute : «Le peuple canadien est libre. Chaque province du Canada est libre. Les Canadiens n'ont pas besoin d'être libérés » (Thompson D., $1990: 270$ ).

Les réactions dans la presse québécoise et canadienne se déchaînent contre le comportement du président français. Si Jean-Marc Léger du Devoir de Montréal qualifie de "paroles de vérités, un message d'espoir » la déclaration de De Gaulle et que Guy Cormier de La Presse de Montréal écrit : «La vie et la liberté étant les deux plus grands biens de ce monde, on pourrait se demander où exactement réside le problème dans une exclamation comme vivre le Québec libre»; les réactions sont pour l'essentiel négatives.

13 Roger Champoux, éditorialiste en chef de la Presse, écrit : «Fierté oui! Agitation, non! ». Le quotidien le Soleil de Québec souligne que le "Vive le Québec libre» est non seulement le cri de ralliement du Rassemblement de l'Indépendance nationale, un parti indépendantiste assez marginal, mais est aussi à l'origine des « actes de terrorismes qui se sont produits dans la province ». Dans un quotidien canadien d'importance, le Toronto Star, on peut lire: « Nous devons tenir pour acquis qu'il a délibérément offensé et insulté le Canada. Si Lester Pearson avait la moitié de la fierté nationale des Canadiens français, il lui conseillerait de quitter le Canada immédiatement» (tiré de Thompson D., $1990: 271$ ).

14 Mais que pense le Premier ministre Daniel Johnson du discours de De Gaulle? Il faut se rappeler que Johnson, lors d'un séjour à Paris,avait exprimé au président français que le Québec avait besoin de lui, que c'était «maintenant ou jamais». Pour Johnson, le représentant de la France avait un rôle historique à jouer. Lorsque le Général lance à 
Daniel Johnson quelques minutes après son discours: «Je crois que je vous ai embêté, M. le Premier ministre", Johnson répond: "Vous avez lancé le slogan d'un parti adverse » pour ensuite adoucir son discours et dire: « Mais quant au reste ne vous en faites pas, je vais me débrouiller " (Godin P., 1991: 271).Après une réaction initiale plutôt négative, Johnson se ravise et déclare :

Il (de Gaulle) a salué cette conviction qui est de plus en plus celle du peuple québécois qui est libre de choisir sa destinée et que, comme tous les peuples du monde, il possède le droit incontestable de disposer de lui-même, en déterminant librement son développement économique, social et culturel... Le Québec n'a jamais été une province comme les autres. Ce fait élémentaire que d'aucuns au Canada trouvent encore difficile à accepter, le monde entier maintenant le connaît.(Patry A., $1980: 103)$.

\section{La réaction des Québécois}

Un sondage effectué à la fin du mois de juillet et au début du mois d'août 1967 démontre que $60 \%$ des Québécois désapprouvent la déclaration du Général, mais que $40 \%$ y sont favorables. La plupart des Québécois ne prennent pas cette déclaration avec autant de sérieux que la presse canadienne anglophone. Certains sont même ravis que le Général ait remis les « Anglais à leur place » (Thompson D., 1990 : 296-297).

Le chef de l'opposition, Jean Lesage, du Parti libéral du Québec, demande à ses députés de dénoncer la déclaration du président français. Cette position de la part du chef de l'opposition provoquera une démission au sein de son parti. En effet, le député François Aquin démissionnera du caucus du Parti libéral pour siéger comme indépendant, mais aussi comme indépendantiste, à l'Assemblée nationale à Québec. Pour expliquer son geste,François Aquin déclare que Charles de Gaulle a compris :

les aspirations profondes du peuple québécois, il a saisi le tréfonds du drame vécu par nos compatriotes qui sont pauvres dans un pays riche, citoyens de seconde classe dans leur propre cité, forcés de travailler dans la langue des maîtres, étrangers sur le sol même de leur patrie, déchirés entre ce qu'ils sont et ce qu'ils voudraient être ». Quand de Gaulle a lancé son "vive le Québec livre», poursuit Aquin «c'est l'âme de tout un peuple opprimé et brimé qu'est montée spontanément comme une réponse l'acclamation triomphale du 24 juillet». Aquin conclut son discours par les mêmes mots que le Général : «vive le Québec libre ». (Thompson D., 1990 : 296-297).

Probablement influencé par les réactions virulentes de la presse canadienne anglophone du Canada, un nouveau sondage, publié le 12 août 1967 par la maison de sondage CROP, révèle que l'opinion publique québécoise a changé concernant la déclaration du général de Gaulle. Une majorité de Québécois est satisfaite du «vive le Québec libre » du général de Gaulle.

Sentant qu'il pourrait isoler le chef de l'opposition, Daniel Johnson dépose une motion à l'Assemblée nationale du Québec remerciant le général de Gaulle d'être venu au Québec et blâmant le gouvernement du Canada pour son « intrusion » qui a interrompu le voyage du président français. Jean Lesage, coincé, car il avait sévèrement critiqué les propos du président français, propose même qu'on ajoute au texte de la motion : « Nous lui en sommes profondément reconnaissants » (Thompson D., 1990 : 296-297)!

Daniel Johnson déclare finalement le 30 août 1967, à l'Assemblée nationale, que les Québécois n'oublieront jamais "qu'en des mots qui touchent le cœur de tous", de Gaulle avait «évoqué le problème de l'identité distincte du Québec et son immense 
effort d'affirmation » (Thompson D., 1990 : 296-297). Selon Johnson, le Québec venait de vivre un épisode historique en rompant avec deux siècles d'isolement. De plus, cette déclaration du président de Gaulle allait dans le sens de l'action gouvernementale en faveur de l'adoption d'une nouvelle constitution plus conforme à la dualité canadienne et à la théorie des deux peuples fondateurs.

\section{Conclusion}

Le 24 septembre 1968, après une convalescence de plus de deux mois à la suite d'un malaise cardiaque, et alors qu'il se savait gravement malade, Daniel Johnson accordera sa dernière conférence de presse. La question des relations du Québec avec la France refait surface. Le président français venait une nouvelle fois de faire des allusions au régime fédéral canadien en des termes peu élogieux. En réponse à une question des journalistes, Daniel Johnson se contente de déclarer: "Le Général peut avoir ses opinions sur la fédération canadienne. Nous avons les nôtres. Le problème va se régler non pas en France (...) il va se régler au Canada » (Thompson D., 1990 : 296-297). Lors d'une seconde question, Daniel Johnson optera, comme il le faisait fréquemment, pour l'attitude plus conciliante et défendra le général de Gaulle et sa déclaration spectaculaire sur le balcon de l'Hôtel de Ville de Montréal. Johnson déclare en substance qu'en une seule phrase, le Général avait réparé toutes "les omissions d'Ottawa et de son service des Affaires extérieures qui n'a jamais, depuis deux cents ans, représenté le Canada comme un pays à deux cultures» (Thompson D., 1990 : 296-297). Daniel Johnson décède le 26 septembre 1968.

21 Le général de Gaulle fait savoir par l'entremise du Consulat de France à Québec qu'il assistera aux funérailles nationales avec l'accord du gouvernement du Québec. De Gaulle, pour qui le décès de Johnson est " une chose effroyable ", veut déroger à la règle selon laquelle un chef d'État de son rang n'assiste qu'à des funérailles de chef d'État. Cette transgression témoigne une fois de plus de l'amitié de De Gaulle pour Johnson et le Québec. Mais à Québec on s'interroge sur l'opportunité de la venue du président. Pour André Patry, le chef du protocole du gouvernement du Québec, la polarisation de l'opinion publique au sujet des déclarations du Général sur le balcon de l'Hôtel de Ville de Montréal n'augure rien de bon. Il voulait éviter que de Gaulle soit accueilli par des huées en sol québécois. De plus, le rang protocolaire du Général est tellement important qu'il faudra nécessairement lui accorder préséance sur Pierre Trudeau, le nouveau premier ministre du Canada, car au Canada le chef de l'État est le Gouverneur général, le représentant de Sa Majesté la Reine Elizabeth II. Les règles élémentaires, en termes de protocole, l'exigent. Le chef du protocole du Québec fait savoir à l'Élysée que la présence du Premier ministre Couve de Murville serait en soit un très grand honneur pour le gouvernement du Québec.

De Gaulle ne comprend pas ce refus de la part du gouvernement du Québec. Attristé par ce qu'il considère être un nouveau recul de Québec, il adresse tout de même le télégramme suivant à Mme Johnson: "C'est avec la plus profonde émotion que j'apprends la nouvelle du très grand chagrin qui vous frappe. J'avais, comme vous le savez, une exceptionnelle estime et une très vive amitié pour M. Daniel Johnson. Ma femme et moi nous vous prions, ainsi que les vôtres, d'agréer nos condoléances les plus sincères » (Godin P., 1991). 


\section{BIBLIOGRAPHIE}

Godin, Pierre, La difficile recherche de l'égalité, Montréal, Boréal, 1991.

Paquin, Stéphane (dir.), Histoire des relations internationales du Québec, Montréal, VLB éditeurs, 2006.

Patry, André, Le Québec dans le monde, Montréal, Leméac, 1980.

Peyrefitte, Alain, De Gaulle et le Québec, Montréal, Stanké, 2000.

Thompson, Dale C., De Gaulle et le Québec, Montréal, Éditions du Trécarré, 1990.

\section{AUTEUR}

\section{STÉPHANE PAQUIN}

Stéphane Paquin (doctorat Sciences Po Paris) est professeur au département de science politique de l'UQAM. Il est directeur du Groupe de recherche et d'études sur l'international et le Québec (GERIQ) et directeur de recherche au Centre d'études sur l'intégration et la mondialisation (CEIM). Il est également co-directeur de la collection « Politique mondiale » aux presses de l'Université de Montréal. UQAM. paquin.stephane@uqam.ca 